\title{
Enhanced Collision Avoidance for Distributed LTE Vehicle to Vehicle Broadcast Communications
}

\author{
Jianhua He, Zuoyin Tang, Zhong Fan and Jie Zhang
}

\begin{abstract}
In this paper we investigate the distributed autonomous resource selection for LTE vehicle to vehicle (V2V) broadcast. The effectiveness of collision avoidance and location based resource allocation enhancements is examined. It is found that collision avoidance with multiple data resources reservation per schedule assignment (SA) is a key to improve broadcast reliability. However in the existing collision avoidance algorithm reserving multiple resources per SA can lead to many data packet collisions if a SA collision happens. We propose an enhanced collision avoidance to address this issue. The idea is to use selected data packets to disseminate the reservation of data resources and SA resources, which can provide better communication among neighbor vehicles on resource reservation and reduce data collisions. Simulation results show that the proposed collision avoidance enhancement can effectively improve SA and data transmission reliability. The network capacity in terms of supported vehicles under given $\mathrm{V} 2 \mathrm{~V}$ service requirements is largely increased by $17 \%$ at a negligible cost of added overhead.
\end{abstract}

Index Terms-LTE, vehicle to vehicle (V2V), connected vehicles, resource scheduling, semi-persistent scheduling.

\section{INTRODUCTION}

With fast development of autonomous driving connected vehicles is receiving revived interests from industry, which holds great potentials on building cooperative context awareness and cooperative driving. While IEEE $802.11 \mathrm{p}$ has been specified for $\mathrm{V} 2 \mathrm{~V}$ and vehicles to roadside infrastructure (V2I) data exchange [1] [2] [3], the 3GPP specification of LTE network support for vehicle to everything (V2X) services promises better $\mathrm{V} 2 \mathrm{~V}$ performance in terms of reliability, latency and spectrum efficiency [4], [5].

According to the Release 14 of LTE standards, V2V services can be supported over PC5 interface through either centralized scheduling by the eNodeBs or distributed scheduling by the vehicles [4], [5]. Centralized broadcast scheduling can have very high broadcast reliability, but it has a large signalling overhead on vehicle position update and resource allocation [6]-[9]. Distributed scheduling with autonomous resource selection is more scalable and can work when eNodeB cover is not available. Performance gains with collision avoidance (CA) and location based resource allocation enhancements were reported for autonomous resource selection in [4], [10]. However, there enhancements have many design options and parameter settings, the impact of which have not been well studied in the literature [4], [5], [9], [10].

As the quality of $\mathrm{V} 2 \mathrm{~V}$ services is critical for cooperative safety applications, solid investigation and enhancement of

Jianhua He and Zuoyin Tang are with School of Engineering and Applied Science, Aston University, UK, \{j.he7, z.tang1\}@aston.ac.uk. Zhong Fan is with School of Computing and Mathematics, Keele University, UK, z.fan@keele.ac.uk. Jie Zhang is with Department of Electronic and Electrical Engineering, University of Sheffield, UK, jie.zhang@sheffield.ac.uk. Z. Tang is Corresponding Author.
LTE V2V services are needed. The unique features of LTE supported $\mathrm{V} 2 \mathrm{~V}$ services such as synchronized frame structure also offers opportunities for innovative algorithm design. In this paper we first examine the effectiveness of the enhancements with $\mathrm{CA}$ and location based resource allocation, and the impact of the associated design parameters such as the number of resource reservations and SA retransmissions. We find that reserving multiple data resources per SA is the key of improving transmission reliability, while improvement with driving direction base resource allocation is very small. The improvement with reservation of multiple resources per SA can be explained by the largely reduced SA channel loads, which helps reduce SA and data packet collisions.

However, a large issue with multiple resources reservation identified in the existing CA algorithm is that, one SA collision could lead to a large number of data collisions. We believe better communication on resource reservation among neighbor vehicles is feasible and could be exploited to solve the issue. In this paper we follow this direction and propose an enhanced CA algorithm to address the issue of SA collision. Better communication is provided by using some data packets to help disseminate the reservation of data and SA resource reservation. Therefore neighbor vehicles can have an improved view to the reserved resources and reduce collisions. A system level simulator is developed to evaluate and compare several LTE V2V broadcast schemes with different CA algorithms. Simulation results show that the proposed CA enhancement can effectively improve SA and data transmission reliability. The network capacity in terms of supported vehicle density under given $\mathrm{V} 2 \mathrm{~V}$ service requirements is increased by $17 \%$ at very low cost of added transmission overhead. Our work demonstrates the large potentials of vehicle communication.

\section{System BACKGROUND AND Assumptions}

In this paper we consider autonomous resource selection in LTE V2V for which infrastructure support is not required. There are $N_{v}$ connected vehicles in the network. Vehicles broadcast cooperative awareness message (CAM) data packets directly to their neighbors. Data packets are assumed to be generated with a period of $T_{p} \mathrm{~ms}$. The data packet carries the host vehicle's information such as speed, heading, brake status as well as security overhead, which are used to build cooperative awareness and support cooperative safety applications. Each data packet is assumed to have an overall length of 190 bytes. The latency requirement for data packets is denoted by $T_{d}$ (set to 100) in ms.

The connected vehicles are all equipped with one transmission antenna that operates in half-duplex mode. The uplink PHY and MAC specifications of 3GPP LTE are used for 
broadcast communication. Transmissions follow synchronized LTE frame structure with $10 \mathrm{~ms}$ frames and $1 \mathrm{~ms}$ subframes. Each subframe has two time slots of $0.5 \mathrm{~ms}$. Spectrum resource is divided into subcarriers of $15 \mathrm{KHz}$. The minimal unit of resource block $(\mathrm{RB})$ for resource allocation has 1 time slot and 12 consecutive subcarriers in frequency. The resource blocks are organized into fixed size SA and data subchannels for transmission of SA and data packets, respectively. Each data packet has to be scheduled by one SA. The number of RBs included in SA and data subchannels depends on the size of SA and data packets, and the used modulation and coding schemes (MCS). In this paper, we assume one SA subchannel includes $1 \mathrm{RB}$ and one data subchannel includes $14 \mathrm{RB}$. Each transmission resource for a SA and a data packet has one SA and data subchannel over one subframe, respectively. Let $N_{s a}$ and $N_{d t}$ denote the number of SA and data transmission resources in one subframe, respectively, which can be determined according to the system bandwidth, MCS and packet sizes [4] [9]. An example of frame structure and resource organization is shown in Fig. 1, where system spectrum resource is divided into SA and data resource pools with $4 \mathrm{SA}$ resources and 2 data resources in one subframe.

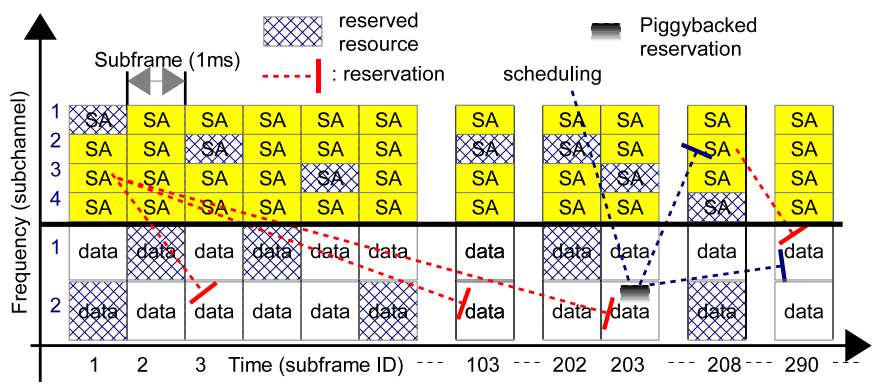

Fig. 1. Illustration of frame structure and resource organization, with 4 SA resources and 2 data resources in one subframe.

\section{Autonomous Selection with Collision AVOIDANCE}

\section{A. Basic Collision Avoidance Algorithm}

According to [4], CA enhancement to the LTE D2D sidelink resource allocation provides gains for LTE V2V distributed broadcast. In the CA algorithm there are two closely coupled processes, namely resource selection and resource reservation. Vehicles keep monitoring the channel, receiving SA and data packets from neighbor vehicles. The time and frequency location of the selected data transmission(s) can be extracted from successfully decoded SAs. We assume that SA and data packets from a single vehicle are transmitted in different subframes. Receiving vehicles set the resources selected by the decoded SAs as reserved. When a vehicle has data packets to transmit, it selects only from the unreserved resources. As safety data messages are usually periodical, vehicles can select and reserve data resources for multiple data packets in one selection process. Then a SA packet is sent over the SA subchannel to announce the reservation of data resource(s).

\section{B. Proposed Enhanced Collision Avoidance Algorithm}

CA with reservation of multiple resources has a large adverse impact As SAs are transmitted randomly without resource reservation, they are prone to collisions. When SA collision happens, neighbor vehicles will not be aware of the resource reservation carried by the collided SAs. The neighbor vehicles may select the same resources reserved by the collided SAs, which is very likely to happen under high channel load. Therefore a batch of consecutive data packets will collide.

As consecutive data packet collisions are highly undesirable for road safety applications, it is critical to reduce SA collisions and mitigate the impact of SA collisions if they happen. However, due to the distributed broadcast nature and halfduplex operation, a vehicle can't detect its own SA collisions and reselect data resources. While neighbor vehicles may cooperative on detecting SA collisions, it is quite complex for a vehicle to choose and inform which neighbor vehicle to help on this task. Next we propose simple but effective enhancements to $\mathrm{CA}$, which are presented below.

1) SA Scheduling: Before schedule data transmissions a vehicle needs to schedules SA transmission(s) first. Let $T_{\text {curr }}$ denote the time (sequence number of subframe) when the scheduling process starts. Suppose the number of SA retransmissions is $N_{\mathrm{rt}}$. The first $\mathrm{SA}$ is scheduled to a SA resource which is selected randomly from the unreserved SA resources within the time window $\left[T_{\text {curr }}+T_{\text {sa,low }}, T_{\text {curr }}+T_{\text {sa,up }}\right]$. $T_{\text {sa,low }}$ and $T_{\text {sa,up }}$ are configurable lower and upper limits of the SA resource selection window, respectively. $T_{\text {sa,low }}$ and $T_{\text {sa,up }}$ are set to 2 and 6 with the consideration of packet preparation and a trade-off in the randomness and transmission latency. The reservation of SA resources is announced through some specific data packets to be introduced later. Once the first SA is scheduled, say at subframe $T_{s a, 1}$, the second SA can be scheduled similarly at unreserved SA resources within time window $\left[T_{s a, 1}+2, T_{s a, 1}+6\right]$. The above SA scheduling process is repeated, until the $N_{\mathrm{rt}}+1 \mathrm{SA}$ (one original SA and $N_{\mathrm{rt}}$ retransmissions) are scheduled. If in the process of scheduling a SA there is no unreserved SA resource in the corresponding time window, we use the next SA resource selection window to schedule this SA. One constraint on the SA scheduling is that the SAs are not scheduled to beyond subframe $T_{\text {curr }}+T_{d} / 4$, in order to leave sufficient resources for data packets selection. Let $N_{\text {sch }}$ denote the number of SA transmissions that are successfully scheduled. Let $T_{s a, n}$ and $B_{s a, n}$ denote the ID of the subframe and subchannel of the resource reserved for the $n$th SA packet transmission, $1 \leq n \leq N_{\text {sch. }}$

2) Data Packets Scheduling: Data packets are scheduled after the last scheduled SA retransmission. For each data scheduling process, the number of data resources to be reserved (denoted by $N_{\text {rsv }}$ ) is randomly selected from [ $N_{\text {low }}$, $N_{\text {up }}$ ]. $N_{\text {low }}$ and $N_{\text {up }}$ are configurable parameters, which are set to 5 and 10, respectively. As we have assumed that the last SA retransmission is scheduled at subframe $T_{s a, N_{\text {sch }}}$, the first data packet is scheduled to a resource from the set of unreserved data resources in the time window $\left[T_{s a, N_{s c h}}+\right.$ $\left.2, T_{\text {curr }}+T_{d}\right]$. Once the first data transmission is scheduled, the remaining data transmissions are scheduled similarly.

3) Resource Reservation: In the basic CA algorithm, the information of reserved data resources is carried by SAs and announced to neighbor vehicles. Vehicles receiving the SAs update their data resource reservation table accordingly. 
Collision avoidance is only applied to data transmissions.

In our proposed enhancements we apply CA to both data and SA transmissions, as SA transmission reliability has a large impact on data packet reception. Each vehicle maintains a table for SA and data resource reservation separately. As done in the existing CA algorithm, data resource reservation information is sent with the SA packets. In addition, as the reliability of data packets with resource reservation is high, we propose to piggyback the information of both SA and data resources reservation by the vehicles in their own data packets. We assume that the radio layer can schedule and request safety messages from the safety application layer. So we can start a new round of resource scheduling just before the subframe where the last data packet is scheduled in the previous round of scheduling. The new round of scheduling follows the aforementioned operation.

Once the SA and data packets for the new round are scheduled, the information of reserved data resources and SA resource for the first SA are sent with the last data packet scheduled in the previous round of scheduling. In this way neighbor vehicles have better chance of tracking the reserved $\mathrm{SA}$ resources and can reduce SA collisions. In addition, they can receive data resource reservation information from the received data packets, largely reducing data packet collisions. It is noted that to reduce the added overhead, only the last data packet scheduled in a SA is chosen to carry the data and SA resource reservation information. As the reserved data resources are usually periodical, a compact representation of the reserved resources can be used, which includes information of the subframe and subchannel of the first reserved data resource, the message periodicity and the number of reserved data resources. Two additional bytes could be sufficient for an efficient encoding of the data resource reservation information. For data packets of 190 bytes and on average 7.5 data resources reservation per SA, the added two bytes control information has only $0.14 \%$ overhead. It is noted that the data packets are scheduled with latency constraint. There is no added latency to the data packet transmissions due to the proposed CA process.

Fig. 1 shows an example operation of the enhanced CA. In the first subframe, a vehicle sends a SA, which carries the information of three reserved data resources at subchannel 2 and subframes 3, 103 and 203. Rightly before subframe 203 where the last data packet is scheduled, the vehicle starts a new scheduling with the next SA scheduled at subframe 208 and the next data packet scheduled at subframe 290. The new reservation information is piggybacked to the data packet scheduled at subframe 203, which gives extra notifications to neighbor vehicles of resource reservation.

\section{EXPERIMENT SETUP AND Simulation Results}

\section{A. System Setting}

We developed a system-level simulator to evaluate the proposed CA algorithm for distributed LTE V2V broadcast under highway scenarios. For each simulation 200 seconds (200 thousands subframes) are simulated. 10 simulations are run to obtain an averaged result. Main simulation parameters are set according to [4] and [11] as shown in Table I. Vehicle density varies from 150 to 210 vehicles per $\mathrm{km}$. Vehicles are randomly dropped to the road with uniform distribution. Data packet generation period is $100 \mathrm{~ms}$. The target packet reception distance is $320 \mathrm{~m}$, and the target average packet reception ratio (PRR) in the range $(300,320) \mathrm{m}$ from transmitters is $90 \%$.

TABLE I

SYSTEM PARAMETERS.

\begin{tabular}{l|l|l|l}
\hline Parameter & Value & Parameter & Value \\
\hline Carrier freq. & $5.9 \mathrm{GHz}$ & Bandwidth & $10 \mathrm{MHz}$ \\
\hline SA resources & $5 /$ subframe & data resources & $2 /$ subframe \\
\hline Antenna height & $1.5 \mathrm{~m}$ & Antenna gain & $3 \mathrm{dBi}$ \\
\hline TX Antenna & 1 & RX Antenna & 2 \\
\hline Tx power & $23 \mathrm{dBm}$ & Noise figure & $9 \mathrm{~dB}$ \\
\hline Shadowing & Log-normal & Std. dev. & $3 \mathrm{~dB}$ \\
\hline Data MCS & QPSK, Turbo 1/2 & Road length & $2000 \mathrm{~m}$ \\
\hline Number of lanes & 6 & Lane width & $4 \mathrm{~m}$ \\
\hline Vehicle speed & $70 \mathrm{~km} / \mathrm{h}$ & Speed std. dev. & $5 \mathrm{~km} / \mathrm{h}$ \\
\hline
\end{tabular}

Four baseline distributed broadcast schemes are compared, including random resource selection (RRS) scheme [10], basic CA supported (BCA) scheme, BCA with location based resource allocation (BCA-L) scheme, and semi-persistent scheduling (SPS) scheme [5]. With the RRS scheme channel is not monitored. A user selects data resources randomly from the set of resources that satisfy the data latency requirement. Such scheme is suitable for transmissions from pedestrians or cyclists with power consumption concern. With the BCA scheme vehicles select unoccupied data resources and update resource reservation according to the decoded SAs. In the BCA-L scheme driving direction based resource allocation is added to the BCA scheme. The whole SA and data resources are evenly divided to two sets, each for vehicles driving in one direction. SPS scheme was adopted in the 3GPP LTE V2X standard [5]. Following [5] data resources are selected from unoccupied resources according to channel sensing in the last $1000 \mathrm{~ms}$ period. A random number (in a range of 5 to $10 \mathrm{in}$ our simulations) of data resources are selected and reserved from available resources in each selection process. The data resources could be retained without a reselection process with a probability $p$, which is set to 0.4 in our simulations.

\section{B. Simulation Results}

The performance metrics of interest include PRR and network capacity. For a packet with ID $n$, the average PRR of this packet over a given range is calculated by the ratio of the number of vehicles successfully receiving the packet (denoted by $V_{s u c, n}$ ) to the number of vehicles (denoted by $V_{n}$ ) in the range. Suppose $N_{t x}$ packets are transmitted in a simulation, then the network wide average PRR for that range is computed by $\sum_{n=1}^{N_{t x}} V_{s u c, n} / \sum_{n=1}^{N_{t x}} V_{n}$.

Firstly we compare the PRR of data and SA packets of the broadcast schemes at different transmitter ranges. Several test profiles are created, which have different settings on the SA retransmission number and/or number of data resources reserved with one SA. Due to space limitation only typical results with two test profiles are presented: Profile 1 is configured with one SA retransmission and one data resource reservation per SA, and Profile 2 is with two SA retransmissions and a random number of data resources reserved in the range $[5,10]$ per SA. 
Fig. 2(a) and Fig. 2(b) present data and single SA PRR results with Profile 1 against transmitter ranges, respectively. A number (say $d$ ) in $\mathrm{x}$-axis indicates a transmitter range $(d-25$, d). Fig. 2(c) and Fig. 2(d) show the results with Profile 2. Only results with vehicle density of 200 are presented. Results with other vehicle densities show very similar performance trends. The broadcast scheme with proposed piggyback based CA is denoted by PCA. The proposed algorithm can work with other enhancements, such as location based resource allocation, which is denoted by PCA-L. The link layer communication performance of data PRR against SNR presented in [11] is used in simulations. The link model PRR against transmitter range is included in Fig. 2, which represents data PRR upper limit for the broadcast schemes without interference or collisions. Note that as SA packets are sent with a more reliable MCS scheme, SA transmissions have a $3 \mathrm{~dB}$ performance boost and SA PRR can be higher than link model data PRR.

Comparing the Profile 1 and Profile 2 results in Fig. 2, we observe that the RRS scheme has the worst performance. Reserving multiple data resources per SA packet improves data and SA PRR for CA based schemes. The large SA PRR improvement shown in Fig. 2(b) comes from the largely reduced SA channel load (more than five folds reduction), which leads to large data PRR improvement in Fig. 2(a). In the studied scenarios, driving direction based resource allocation shows very little improvement when multiple reservations per SA is used. Compared to the SPS and BCA schemes, the proposed schemes with both multiple resource reservation and piggybacked reservation achieve good PRR performance gains for both SA and data. Data PRR improvement of schemes PCA and PCA-L over BCA and BCA-L is around 5\% consistently for transmitter distance beyond 250 meters in Profile 2.

Data PRR improvement has large impact on the LTE V2V services as V2V safety applications have strict PRR requirements. Next we examine the network capacity with the CA supported broadcast schemes. Network capacity is defined by the maximal vehicle density, with which the target safety application requirements can be satisfied (i.e., 90\% average PRR at 320 meter transmitter distance). To find the network capacity, data packet PRR in range $(300,320)$ meters against vehicle density is shown in Fig. 3(a) and Fig. 3(b) for Profile 1 and Profile 2, respectively. Clearly broadcast schemes with Profile 1 has network capacity of less than 150. Schemes with Profile 2 have much higher network capacity. According to Fig. 3(b), the proposed schemes PCA and PCA-L have network capacity of 193 and 200 (vehicles $/ \mathrm{km}$ ), respectively, while BCA and BCA-L have capacity of 165 . There is a remarkable network capacity gain of $17 \%$ and $21 \%$ respectively for schemes PCA and PCA-L over BCA and BCA-L.

It is noted that the data PRR and network capacity gains of the proposed schemes come mainly from the piggybacked data resource reservation information, which are verified by experiments but the results are not presented here due to space limit. Piggybacking SA resource reservation does improve SA PRR but makes small contribution to data PRR. Therefore Piggybacking only two bytes data resource reservation information for one resource scheduling can considerably improve LTE V2V data reliability and network capacity. The proposed scheme is also backward compatible, as legacy ve- hicles supporting $\mathrm{V} 2 \mathrm{~V}$ can ignore the piggybacked reservation information in received data packets.

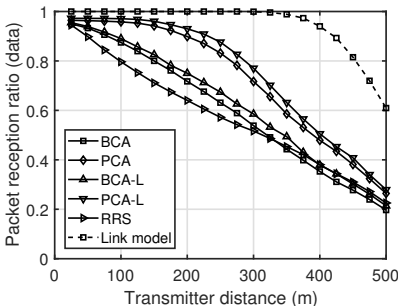

(a) Data PRR with Profile 1.

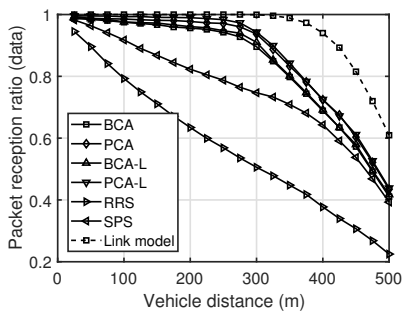

(c) Data PRR with Profile 2.

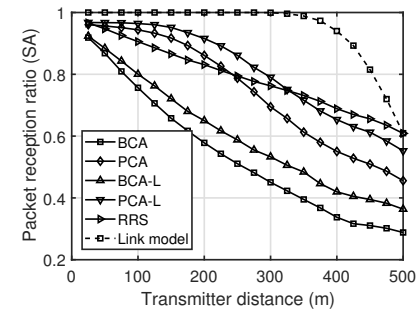

(b) SA PRR with Profile 1

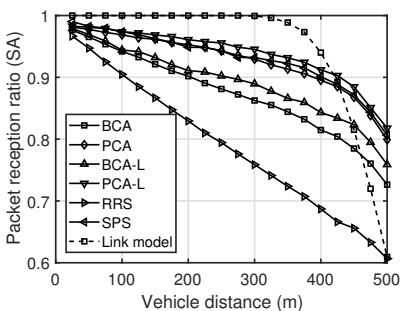

(d) SA PRR with Profile 2
Fig. 2. Average data and SA PRR of broadcast schemes versus transmitter distance. (a) and (b) for Profile 1: one SA retransmission and single data reservation per SA; (c) and (d) for Profile 2: two SA retransmissions and multiple data reservations per SA. Vehicle density is 200 vehicles $/ \mathrm{km}$.

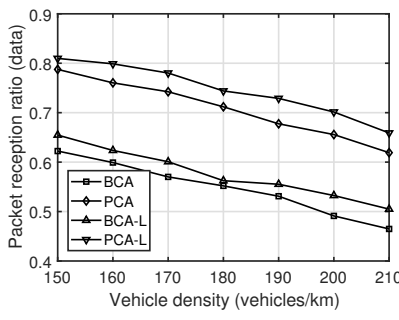

(a) Profile 1.

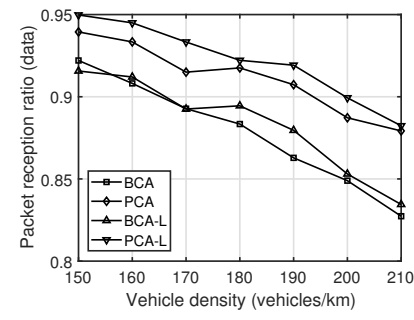

(b) Profile 2
Fig. 3. Data PRR in distance range $(300,320) \mathrm{m}$ versus vehicle density.

\section{REFERENCES}

[1] 'TS 102 687: intelligent transport systems (ITS); decentralized congestion control mechanisms for intelligent transport systems operating in the $5 \mathrm{GHz}$ range; access layer part', ETSI, July 2011.

[2] He J., Chen H., et al, 'Adaptive congestion control for DSRC vehicle networks', IEEE Comm. Lett., Feb. 2010, 14, (2), p.127-129.

[3] Alexey Vinel, '3GPP LTE Versus IEEE 802.11p/WAVE: Which Technology is Able to Support Cooperative Vehicular Safety Applications?', IEEE Wireless Comm. Lett., April. 2012, 1, (2), p.125-128.

[4] "Study on LTE-based V2X services", 3GPP TR 36.885, V14.0.0, 2016.

[5] Chen, S., et al, 'Vehicle-to-Everything (v2x) services supported by LTEbased systems and 5G', IEEE Comm. Std. Mag., June 2017, 1, (2), p.70 $-76$.

[6] Bazzi A., et al, 'On the performance of IEEE 802.11 p and LTE-V2V for the cooperative awareness of connected vehicles', IEEE Trans. Vehicular Tech., 66, (11), Nov. 2017.

[7] Di B., et al, 'Non-orthogonal multiple access for high-reliable and lowlatency V2X communications in 5G systems', IEEE JSAC, July 2017.

[8] Zhang X., et al, 'Research on overlay D2D resource scheduling algorithms for V2V broadcast service', IEEE VTC, Sept. 2016.

[9] Cecchini, G., 'LTEV2Vsim: An LTE-V2V simulator for the investigation of resource allocation for cooperative awareness', IEEE Conf. Models and Technologies for ITS, June 2017.

[10] Yang, J., et al, 'Enhanced autonomous resource selection for LTE-Based V2V communication', IEEE VNC, Dec. 2016.

[11] "Vehicle to Vehicle (V2V) services based on LTE sidelink; User Equipment (UE) radio transmission and reception", 3GPP TR 36.785, V14.0.0, Oct. 2016. 